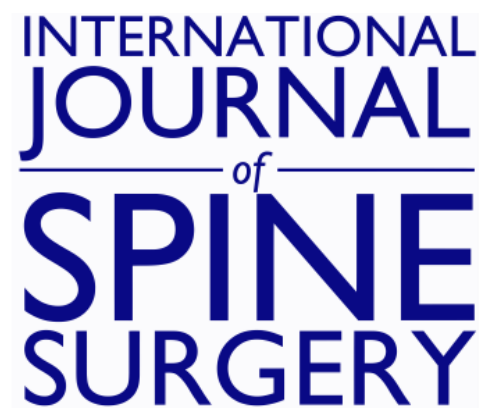

\title{
History and Evolution of Spinal Robotics in Pediatric Spinal Deformity
}

Dennis P. Devito and Raymund Woo

Int J Spine Surg 2021, 15 (s2) S65-S73

doi: https://doi.org/10.14444/8141

http://ijssurgery.com/content/15/s2/S65

This information is current as of April 26, 2023.

Email Alerts Receive free email-alerts when new articles cite this article. Sign up at:

http://ijssurgery.com/alerts

The International Journal of Spine Surgery

2397 Waterbury Circle, Suite 1,

Aurora, IL 60504, Phone: +1-630-375-1432

(C) 2021 ISASS. All Rights,Refserved 


\title{
History and Evolution of Spinal Robotics in Pediatric Spinal Deformity
}

\author{
DENNIS P. DEVITO, MD, ${ }^{1}$ RAYMUND WOO, $\mathrm{MD}^{2}$ \\ ${ }^{I}$ Medical Director, Spine Program, Children's Healthcare of Atlanta-Orthopedics and Sports Medicine, Atlanta, Georgia, ${ }^{2}$ AdventHealth Medical Group, \\ Orlando, Florida
}

\begin{abstract}
Robotic assistance in surgical procedures is a valuable tool that enhances the safety and efficacy of invasive surgeries. These devices are divided functionally into surgeon surrogates where the device operates under the direct control of an offsite surgeon, and surgeon adjuncts where the device is an intraoperative guidance tool used in a portion of the procedure. The current state of robotic spine surgery focuses on the latter, addressing the primary task of pedicle screw placement. We would like to share our experience with the Mazor Robotics devices to discuss the underlying concepts, strengths, weaknesses, and results as they pertain to pediatric spine deformity.
\end{abstract}

Focus Issue Article

\section{CONCEPT}

Robotic-assisted spine surgery utilizes preoperative computed tomography $(\mathrm{CT})$ images of the spine for 3-dimensional (3D) visualization of vertebral anatomy. Visualizing the sectional anatomy in the axial, coronal, and sagittal planes allows the surgeon to understand the pedicle and vertebral body dimensions. The surgeon can then determine the best fitting pedicle screw, the optimal entry point, and the ideal insertion trajectory. This information is saved and downloaded into the robot workstation for real-time stereotactic guidance. The robot then uses image-guided arms for screw placement.

The best asset of any robotic-assisted spine surgery platform is the accompanying software that allows the construction of a preoperative plan from the spine CT. Installed on a laptop computer, the software segments the spine into separate vertebral bodies and consecutively labels each segment. The software removes the spatial misorientation associated with any deformity and reformats the images in proper orthogonal axial, lateral, and coronal planes. The surgeon then selects an easily identified spatial reference point on each $\mathrm{CT}$ plane and biplanar fluoroscopy. By convention, points at the junction of the posterior vertebral wall, the inferior pedicle margin, and the midpoint of the spinal canal's anterior margin are selected. A screw of the optimal length and width is selected using superimposed templates, and the surgeon plans the best entry and pedicle trajectory. The vertebra and implanted screws are then viewed in serial axial, lateral, and coronal slices to verify placement. The final implant construct plan is then reconstructed into a $3 \mathrm{D}$ image of the entire spine, allowing the surgeon to adjust implants to avoid tulip-tab collision, align percutaneous skin incisions, optimize colinear rod reduction alignment, and determine rod length. The surgeon saves the final plan as an exportable file for surgery. ${ }^{1}$

The CT plan is reformatted intraoperatively to match the patient's position on the operating room (OR) table using biplanar fluoroscopy. A reference marker is attached to a known bony landmark, such as a spinous process. Its relationship to a fiducial grid attached to the fluoroscope receiver is captured on an anterior-posterior and an oblique lateral image of the spine. The surgeon marks the anteriorposterior and oblique lateral fluoroscopy images of a chosen vertebral body with the same reference point previously determined on CT. The software uses the superimposed image of the reference marker to the fiducial grid to determine the fluoroscopic beam's orientation in relation to the patient's spine. The software then reformats the CT scan to match each vertebra's position to the patient's spine as positioned on the OR table. The software selects critical landmarks such as vertebral endplates, pedicles, and posterior vertebral margins and rejects image artifacts such as evoked potential 


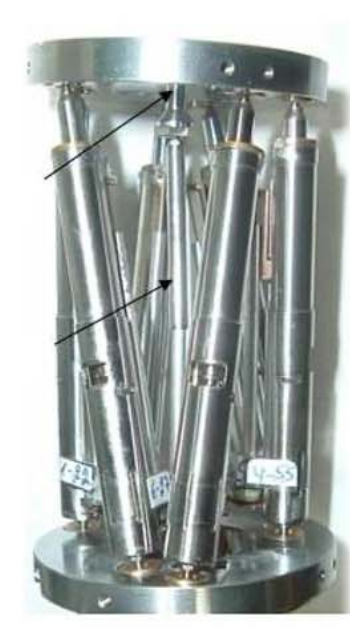

A.

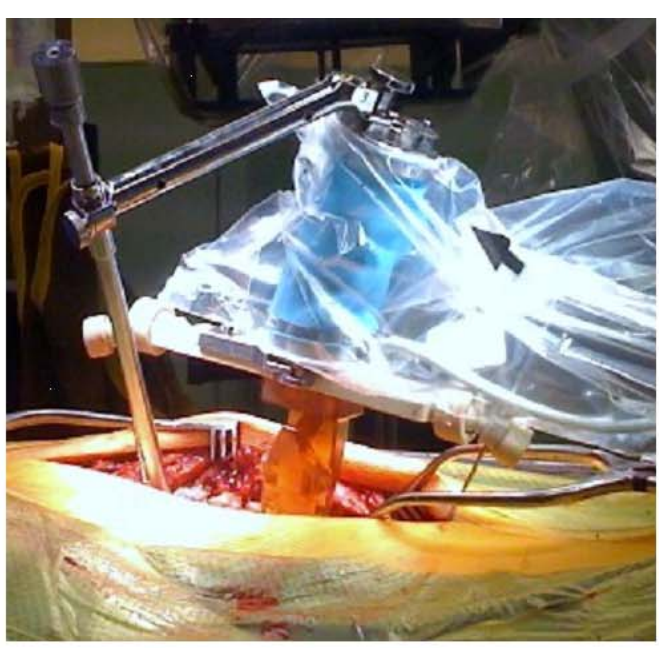

B.

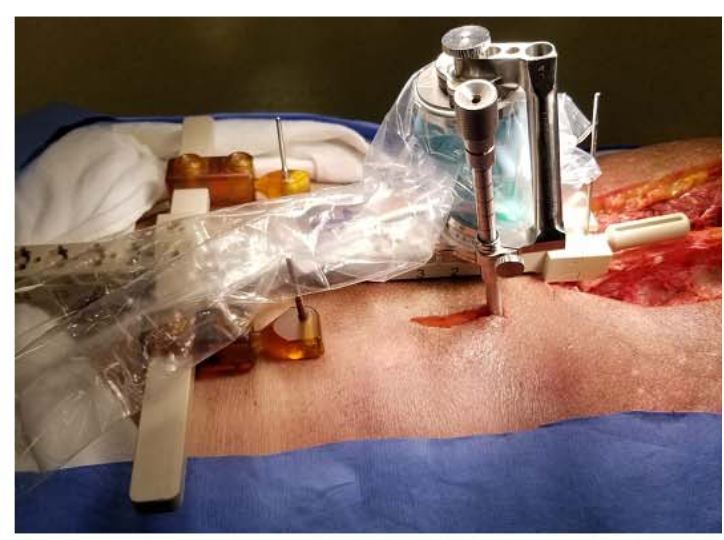

C.

Figure 1. (A) SpineAssist with 6 internal actuators. (B) Robot on spinous process-mounted platform, with arm and drill guide. (C) Hoover-T robot platform mounted on pelvis; with percutaneous drill guide placement.

wires, electrocautery leads, and OR table pads. As compared to conventional surface topography registration, this image registration process is entirely software-based and has a matching tolerance of $1.2 \mathrm{~mm}^{2}$

The first Mazor robotic device is a $50 \times 90-\mathrm{mm}$ cylindrical hexapod device with a base plate and top plate that weighs $400 \mathrm{~g}$. It has 6 actuator struts that move the top plate against the base plate in 4 planes with a motion control resolution of $10 \mu \mathrm{m}$. An effector arm fitted with a drill/tap guide is attached to the top plate. The in vivo targeting accuracy of the drill/tap guide is $<1 \mathrm{~mm}$, including CT and Carm distortion. $^{2}$

The robot's bottom mounting plate mounts to the patient via a 3-position rail attached to the previously placed reference marker. The 3-position rail is best for complex scoliosis cases. Optionally, a T-shaped 14-position rail mounts across the patient's posterior iliac spines and a proximal spinous process, and the reference marker is attached to one of the position stations during image registration. This latter rail is best for longer constructs with minor scoliosis or minimally invasive percutaneous cases (Figure 1).

The robot tethers to a workstation that provides image registration, kinetic calculations, and realtime motion control of the robot. The planned screws are adjusted in real time to accommodate the limits of reach by the robot arm. Although obstruction by the mounting rail and the robot itself limits reach, multiple arm options with a drill/ tap guide on end are available that allow alignment with rigid stereotaxis.

The surgeon cannulates the pedicle with a drill bit guided by the arm's end. A drill stop limits the bit to $24 \mathrm{~mm}$ of depth. Various corded or battery-powered drills can be used, with a reciprocating or constant rotation at the surgeon's desired speed. Using a power drill allows surgeons to use the precise proprioceptive feedback from their fingers and hands to feel the drill bit's advancement through the pedicle. Because little axial pressure is needed to cannulate the pedicle, the surgeon can easily detect the cortical pedicle wall. The surgeon can also easily detect a cortical breach if there is a sudden loss of drill resistance.

The drill bit is then removed and replaced with a passing tube that is slightly larger in diameter than the drill. The surgeon uses the trabecular bony resistance against the tube to verify that it is within the pedicle. The surgeon removes the guide arm, passes a guidewire through the tube into the pedicle, and can note an anterior breach if there is failure to encounter bony resistance when the wire is fully seated. The surgeon taps the pedicle with a cannulated tap and passes a cannulated pedicle screw over the wire. Alternatively, the wire can be removed and replaced with a self-tapping solid screw. The surgeon removes the robot and rail at the end of each registration, and the next series of vertebral bodies are registered.

We use 3 screw placement strategies: (1) drilling all pedicles with guidewire placement for placement of all screws following robot removal, (2) drilling all 
pedicles and marking each hole with bone wax for later screw placement, and (3) drilling and immediately placing a solid or cannulated screw as the robot moves the arm to the next pedicle. The surgeon's choice will vary depending on the deformity and indication. This robotic device's indications for pediatric deformity usually involves open and transmuscular approaches, although percutaneous insertions are occasionally utilized in the lumbar spine and for trauma.

\section{SPINEASSIST (2004-2011)}

SpineAssist was the only spine robot approved by the US Food and Drug Administration during this time. Early adoption was slow, with 3 devices in use in the United States after its introduction in 2004. A neurosurgeon used one device for lumbar degenerative cases to reduce fluoroscopy time. Another was used by a surgeon performing primarily degenerative and a few scoliosis deformity operations on adults. The senior author (D.D.) began using the device in pediatric scoliosis cases in 2006, and by the following year, these 3 surgeons had performed a combined total of 100 cases.

Building on the initial concept, the strengths of SpineAssist were its small footprint and accuracy. Because the device mounts to the spine, patient movement is well tolerated. However, its software was still rudimentary, with difficulty acquiring image registration in spinal deformity cases. These first cases were prolonged and tedious.

The robot's reach was limited by the number of vertebral bodies visualized on the fluoroscopy images, the screw start point and trajectory, the limits of the available arm attachments, the number of available mounting rail positions, and soft tissue pressure against the arm by the wound edges. Soft tissue impingement, especially at the terminal ends of an open incision, significantly contributed to misplaced screws due to lateral pressure against the robotic arm. It was challenging to place retractors within the wound because of interference with the robotic arm's movement. Furthermore, excessive retraction with a hand retractor would cause the patient to shift relative to the robot, increasing the chance for screw misplacement. Drill skive against the lamina and transverse processes also contributed to screw misplacement. Meticulous preparation of the entry sites was needed and best done before the applied rail, as the robot's bulkiness interfered with access with a rongeur.
While the surgeon's overall robotic learning curve is predictable ${ }^{3}$ we were unable to demonstrate any significant learning curve effect. We reviewed the first 50 adolescent idiopathic scoliosis (AIS) patients who underwent robotically guided pedicle screw placement by a single surgeon (R.W.) who had no prior robotic or navigation experience. A modified Gertzbein-Robbins grading system was used by 2 radiologists to study the axial, coronal, and sagittal images on each patient for evidence of medial, lateral, anterior, superior, or inferior cortical breach. ${ }^{4}$ A screw was considered malpositioned if there was a greater than 2-mm breach. Patients were divided into 3 sets and evaluated for the presence of a surgical learning curve by comparing the initial $1 / 3$ of patients with the final $1 / 3$ of patients for presence, degree, and direction of malposition. Initially 50 patients were identified; 2 had postoperative CT studies deemed inadequate for pedicle screw grading. Hence the total number was 48, with 662 pedicle screws. In total, 48 screws $(7.2 \%)$ were malpositioned and the overall screw placement accuracy rate was $92.8 \%$. The most common mistaken direction was laterally $(55.6 \%)$, followed by medial $38.9 \%$, superior $1.9 \%$, and anterior $3.7 \%$. No inferior breach was recorded in any patient. The initial third of patients (16) had a total of 250 screws with $9.6 \%$ of screws malpositioned. The final third of patients (16) had a total of 203 screws with $7.4 \%$ of screws malpositioned. This was not significantly different. Most of the significant breaches were at the incision ends, illustrating the difficulties with soft tissue impinging on the robotic arm. The next most common location was at the concave apical pedicles, where there is often significant pedicular hypoplasia (R. Woo, L. Varich, unpublished data 2014).

Imaging was also a significant challenge for complex deformity cases, particularly the oblique lateral image. Obese or osteoporotic patients posed significant resolution problems. Also, deformities with high pelvic obliquity or a high thoracic curve presented obstruction problems with the iliac wing or contralateral humerus, respectively. Severe scoliosis with curves more significant than 100 degrees and severe rotation posed difficulty in obtaining a clear image of the vertebral endplates or posterior walls. Registration required more images of small segments of the spine, with multiple registrations across the deformity, which can be time consuming. 
Mounting options of the spine were complex in a patient with pronounced spinal deformity. Variation in spinous process shape in severe scoliosis cases makes it difficult to align the rail with the spine. With severely rotated deformities, the spinous processes are very gracile and windswept and can easily break under the clamp. Finally, mounting the device at a lateral angle places an excessive cantilever load on the mount and can cause shifting of the platform or fracture of the spinous process. We prefer the T-rail attachment for severe kyphotic deformities, but its length makes it difficult to mount parallel to the deformity's lordotic and kyphotic segments. Adjustments to the vertical height can accommodate most of these problems, but on obese patients, the longer drill guides are necessary, otherwise the T-rail could be too far away from the spine for adequate reach. Early reports investigating the accuracy of pedicle screws placed with robotic assistance were favorable., ${ }^{2,5}$

\section{RENAISSANCE (2011-2021)}

Using the original SpineAssist robot module, Renaissance was an evolutionary software upgrade with 7 software revisions designed to improve image recognition for registration. Additional features included auto-segmentation of vertebral bodies, vertebral auto-labeling with preservation of screw alignments, faster image processing, and improved artifact rejection. We presented the first multicenter study on adolescent idiopathic scoliosis instrumented with image-guided robotics at the International Meeting on Advanced Spine Techniques in 2014. This retrospective review included 223 patients from 5 centers and demonstrated an overall clinical accuracy of over $99 \%$ after placing 3274 screws. Total robot time averaged 4.5 minutes per screw, with a consistent standard deviation of only 1.5 minutes between surgeons regardless of technique. There were no screw revisions or neurological complications. ${ }^{7}$

Significant technique improvements addressed many earlier concerns. The use of guidewires and cannulated screws reduced skive and is essential in the accurate placement of in-out-in pedicle screws. Also, using a 30.5-cm (12-inch) fluoroscopy receiver significantly reduced registrations by allowing visualization of more segments. We developed exposure and skin incision modifications that decreased soft tissue impingement. The addition of blind screw insertion techniques allowed the percutaneous or transmuscular placement of screws beyond soft tissue retraction limits. The use of power drills for tapping and screw insertion decreased surgeon fatigue and saved time, and adoption of the "drill and fill" strategy for screw placement made use of the time it took for the robotic arm to move between positions. Finally, changes in patient positioning and wire, lead, and electrode placement helped minimize radiographic artifacts in our images.

In 2016, we conducted a 10-year review of Renaissance robotic guidance in order to evaluate efficiency improvements longitudinally, comparing the first 120 patients to the last 50 in a series of over 500 patients with AIS. ${ }^{8}$ We saw significant improvements in the ratio of planned to placed screws and a significant reduction in screw insertions converted to freehand from robotic guidance. Total robot time (including registration time) was reduced in half ( $P$ $<.001$ ), and total operative case times were reduced by an average of 38 minutes $(P=.003)$. Finally, total screw insertion times under robotic guidance decreased from 4.7 minutes per screw to just under 2 minutes, fluoroscopy time during robot use dramatically decreased to only $0.5 \mathrm{sec} / \mathrm{screw}, P<$ .001 . Further analysis of surgical efficiency revealed that measuring triggered electromyography by stimulating inserted pedicle screws wasn't of sufficient value to continue this process, as a result of the high accuracy of robotic placement without the need for redirecting screws. ${ }^{9}$

However, few reports exclusively used CT for assessing screw placement accuracy in pediatric patients. We investigated the correlation between screw accuracy and a supine versus prone preoperative CT for AIS patients undergoing surgery and reported our results using postoperative $\mathrm{CT}$ analysis of screw placement. Of 662 screws evaluated, 48 screws $(7.2 \%)$ demonstrated a breach of greater than $2 \mathrm{~mm}$. With preoperative prone-position CT scanning, we found only $2.4 \%$ of screws to have this degree of a breach. We found medial malposition in $3 \%$ of screws, a rate which decreased to $0 \%$ with preoperative prone-position scanning. In our study, the robot-assisted screw misplacement rate was lower than similarly constructed studies evaluating conventional (non-robot-assisted) procedures. ${ }^{10}$

Such studies in pediatric patients are safely performed if significant measures to reduce radiation dose are implemented. Minimum radiation dosing is essential in pediatric patients requiring a comprehensive thoracic and lumbar spine study, 
with ideal doses less than $1 \mathrm{mSv}$. We reported our experience with a low-dose CT radiation preoperative and postoperative scanning protocol, which yielded an effective dose reduction of $88.0 \%$ for a body mass index (BMI) of $<25$ and $86.9 \%$ for BMI of 25-35. This study demonstrated that sub-millisievert scanning is possible in pediatric scoliosis patients with a BMI under 25 and that larger patients (BMI 25-35) may be scanned very near 1 $\mathrm{mSv}$. This low-dose $\mathrm{CT}$ protocol produces radiation doses that are within the range typical for 2-view scoliosis radiographic examinations of patients aged 13 to 17 years $(0.43-1.63 \mathrm{mSv}){ }^{11}$

\section{MAZOR X AND MAZOR X STEALTH}

In 2016, image-guided robotic surgery went in a slightly different direction with the emergence of the Mazor X (Mazor Robotics) device. Unlike the "miniaturized" Renaissance platform $(50 \times 90$ mm, weighing $400 \mathrm{~g}$ ), Mazor X uses a much larger mechanical surgical arm that requires fewer tool attachments and has more reachable trajectories due to its greater range of movement, thus increasing the work capacity. ${ }^{12,13}$ This robotic arm uses a unique integrated linear optic camera to self-detect its location, creating a volumetric scan of the operative field and thus avoiding collision during movement. This scan uses 5 pictures of the operative field to create a 3D image of the area around the device's attachment to the patient. The larger, more stable Mazor X robotic arm reduces unwanted movement of the drill guide during pedicle preparation. Furthermore, the main frame of the robot is table mounted for more stability while the base of the arm is attached to the patient. This table mount creates a larger footprint than the smaller Renaissance device and cannot be readily removed from the operative field until the completion of the case, although the arm can be swung out of the way for greater exposure of the operative field.

Mazor X uses a modified version of the Renaissance planning software and kinematic calculations for accurate robot arm stereotaxis. The device uses improved image recognition algorithms for fast registration and synchronization of the preoperative CT scan with biplanar intraoperative fluoroscopic images (anterior-posterior and oblique). These upgrades are helpful with complex spinal deformity cases where the identification of individual vertebral levels by fluoroscopy alone can be challenging. Finally, the Mazor X registration process replaces accuracy values averaged from multiple spinal levels by a more precise process where each vertebral level has its specific accuracy.

Surgeons did not widely use Mazor X during its first 2 years of availability. One of the first centers to use Mazor X and create techniques specific for pediatric spinal deformity correction was Children's Healthcare of Atlanta. During this time, approximately 100 consecutive cases of adolescent deformity were instrumented with Mazor X, placing 1608 pedicle screws. Total robot set-up time added approximately 35 minutes to each case, which included applying the custom plastic drape for sterility, registration, and fluoroscopy capture. Robot movement to each subsequent spinal level was noticeably faster with Mazor X than Renaissance. Preparation of the docking site for the drill guide remained a critical step to ensure precision and to minimize skive. Screw placement accuracy remained at over $98 \%$ as determined by intraoperative fluoroscopy and postoperative radiographs using a clinically accepted rating system. Complications included 3 durotomies and 14 wrong drill paths that required manual redirection; however, there were no adverse neurologic events or later screw revisions (D.P. Devito, unpublished data, 2020).

After the purchase of Mazor Robotics by Medtronic, the Mazor X Stealth Edition became available in 2018. This modification integrated Stealth navigation technology with the Mazor X robotic features, enabling real-time visualization of robot-guided trajectories, drilling, and screw insertions. ${ }^{12}$ Mazor X Stealth introduced a new method of registration and planning called "scan and plan." A preoperative CT scan is not required with the scan-and-plan method; however, 3D images of the spine must be captured during surgery by the $\mathrm{O}$-arm (Medtronic), which recognizes the robotic array along with the navigation array. The scan-and-plan workflow obviates the need for any intraoperative fluoroscopy or preoperative CT scan and further reduces operating room clutter. ${ }^{14}$ However, the scan-and-plan process requires implant sizing and trajectory planning to be accomplished while operating, rather than during the preoperative period, which may be a disadvantage. ${ }^{15}$

Robotic technique and workflow for the pediatric patient involve prone positioning with the attachment of the robotic arm on the caudal aspect of the Jackson table. After sterile prep and drape of the 


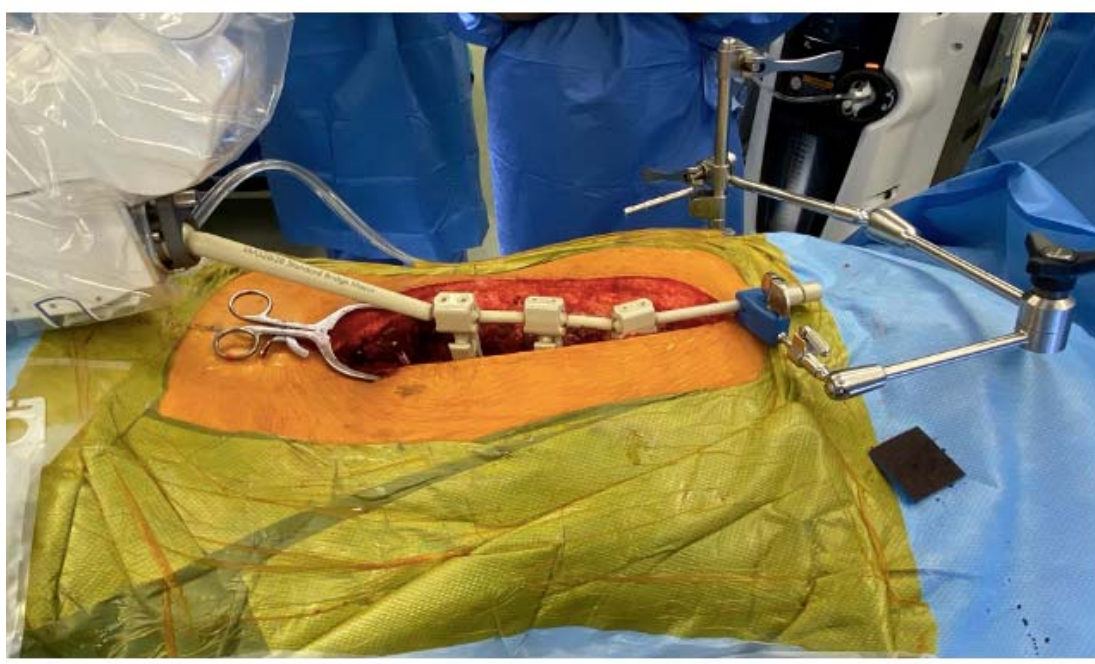

A.
B.

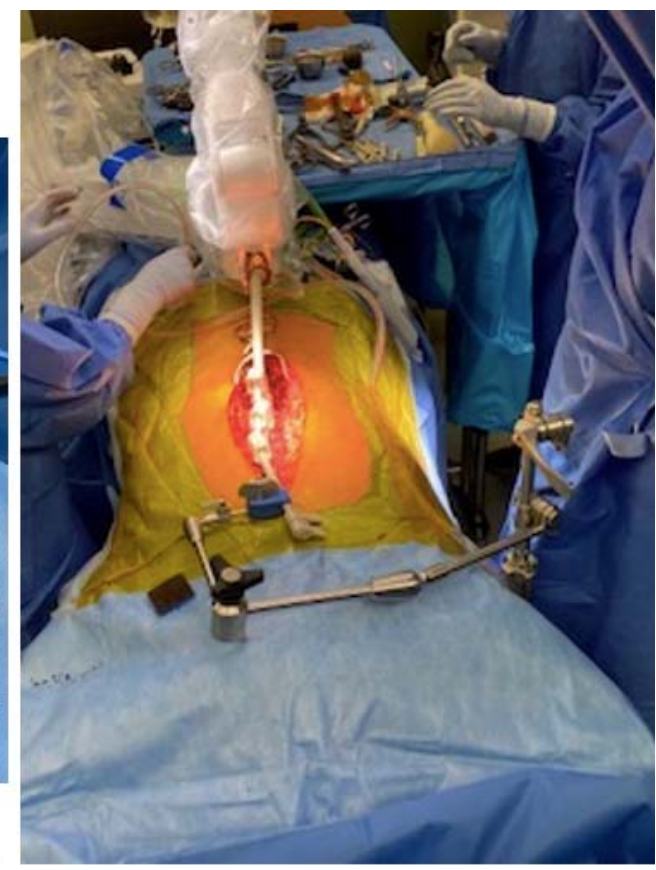

Figure 2. (A) Robotic arm base connected to spinous process-linked clamps; connected to Thompson clamp back to the table. (B) Cephalad to caudal view showing stabilized spine.

patient, the surgeon places a temporary drape on the field. The robotic arm extends over the patient for application of the translucent sterile cover. The end effector is attached to the arm as well as the navigation array; then, the arm is moved off the field and the temporary drape is removed. After exposure of the spine, the robotic arm is again moved into the field and is attached to the patient using either Schanz screws in the pelvis or, preferably, to multiple linked spinous process clamps. The surgeon can further minimize patient spine motion, especially during soft tissue retraction, by connecting the linked clamps back to the OR table (Figure 2). After the robot work area is defined, the registration process is completed either through the scan-and-plan method or fluoroscopic synchronization to the CT scan. The robotic arm moves from point to point, allowing for docking of the drill guide, drilling, and screw insertion through the robotic arm. With the Mazor X Stealth edition, a navigated high-speed burr removes any bone obstructing the planned trajectory path. This new navigated tool eliminates the need to tamp the drill guide onto the bone firmly, and thus skiving is reduced. On average, 6 to 7 spinal levels (12-14 screws) are instrumented in 1 registration before repeating the process if more spinal levels are to be instrumented. With the Mazor X Stealth adoption, our center now demonstrates equivalent total robot usage times compared to Renaissance, despite a slightly longer set-up time. This equivalency is due to faster movement of the robotic arm, and no longer manually moving the robot to a different station or having to change drill guides. Occasionally, real-time adjustment of drill trajectory is required to avoid interference from the spinous process clamps. Earlier studies reported S2 alar-iliac screw placement accuracy with Renaissance to be adversely affected by deflection of the more mobile Renaissance drill guide, ${ }^{15-17}$ even though accuracy was high between $95.7 \%-100 \%$ in some series, with only a 2-mm difference between actual and planned trajectories. ${ }^{18,19}$ However, with the Mazor X's more rigid arm, soft tissue or device-related interference has not been our clinical experience, including the challenging distortions presented by neuromuscular pelvic obliquity (Figure 3).

Although there are published reports on the effectiveness and results of robotic-assisted spine surgery with the Renaissance device, very little information has been reported about Mazor X and its application in pediatric spine deformity. In a recent pediatric cohort study where surgeons placed 314 pedicle screws with Mazor X Stealth, an accuracy of $98.7 \%$, with no screw-related complications, was reported. ${ }^{20}$ Currently, 4 collaborating pediatric centers are investigating robotic-assisted navigation in pediatric patients with this device; 2 


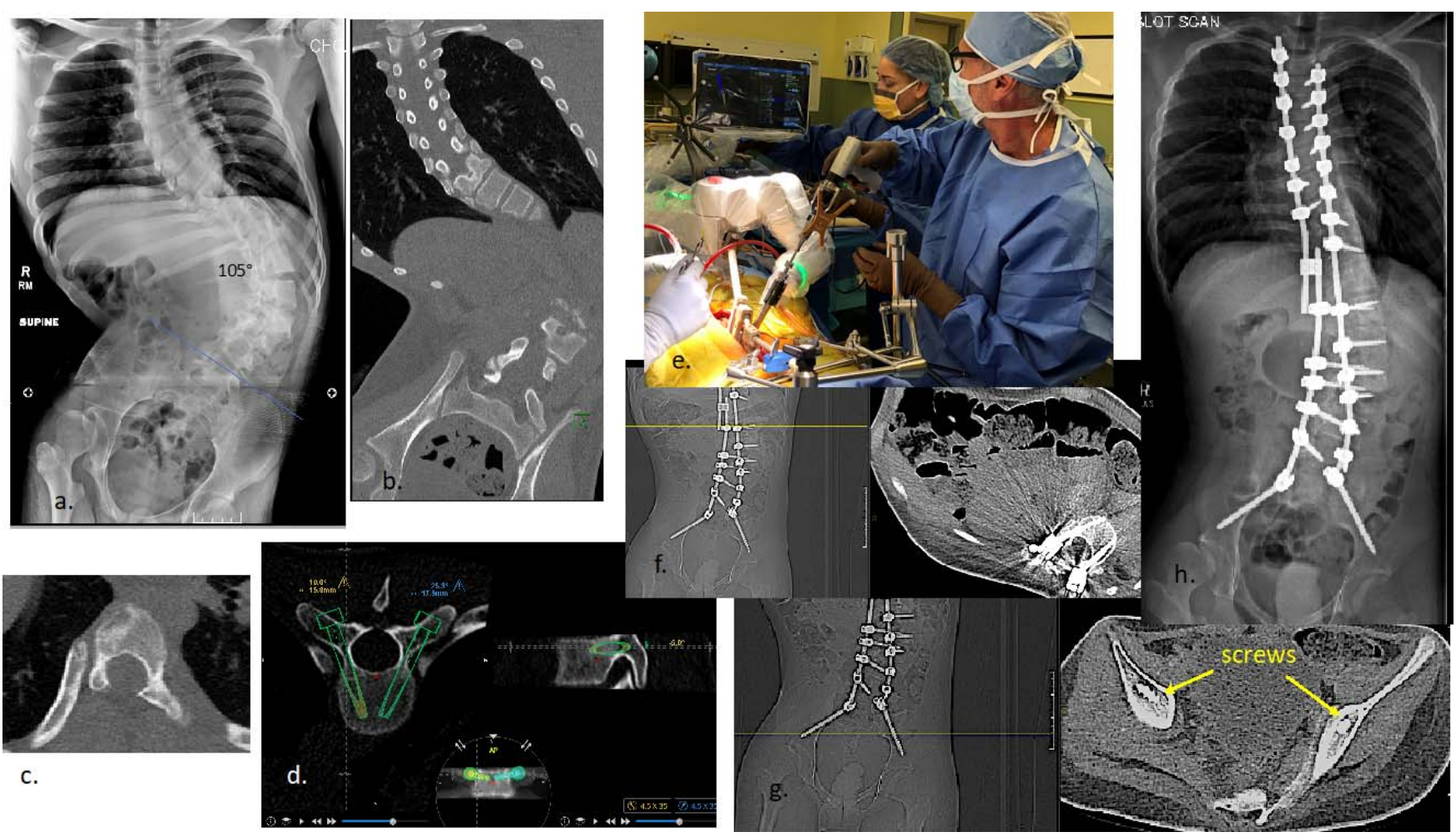

Figure 3. (A) Adolescent with rigid neuromuscular scoliosis with pelvic obliquity induced by post-spinal cord tumor resection. (B) Computed tomography (CT) scan showing pelvic position for planning S2 alar-iliac (S2AI) screws. (C) Absent lamina in region of prior tumor resection. (D) Mazor software with planned screw size and trajectories. (E) Mazor X Stealth robotic alignment and observed navigated drilling of planned screws. (F) Example of thoracic robot-guided screw placement. (G) Accuracy of S2AI robotic screw position verified on postoperative CT scan. $(\mathrm{H})$ Final postoperative alignment.

utilize scan-and-plan workflow, while the other 2 use pre-operative CT and intraoperative fluoroscopic registration (all have the same robotic navigation package). Until those data are collated, we can only draw parallels to prior studies of the effectiveness of this technique. While reproducible accuracy is a recognized goal, we need to also demonstrate improved operating room efficiency, reduced radiation exposure, safe surgery, and ultimately better clinical outcomes. Despite the advantages of this newer technology, several key factors are necessary to integrate the robotic process successfully. The surgeon must understand the mechanical properties of the entire operative procedure and be willing to build confidence and skill through a defined learning curve, after which proficiency leads to more efficiency, as shown with the Renaissance experience. $^{4,9}$

\section{SUMMARY}

While computer-assisted surgical navigation has been an available adjunct for many years to spine surgeons, relative inaccuracy, workflow inefficiency, and high cost have hindered its widespread use. ${ }^{21}$
The adoption by spine surgeons of image-guided robotics, which have been in clinical use for 15 years, has only recently gained momentum as a reliable surgical tool. This momentum comes from the continued push for minimally invasive techniques with limited surgical exposures that require precise implant placement without excessive fluoroscopy. The ability of the software to accurately and quickly register individual vertebrae in patients with pronounced spinal deformity or iatrogenically distorted anatomic landmarks makes this technology also appealing to pediatric deformity surgeons who perform open procedures.

Image-guided robotics have repeatedly shown a high degree of accuracy with implant placement, and this accuracy continues to improve with added techniques to reduce drill-bit skiving and soft tissue impingement against the device. Patient safety, which is empirically directly correlated to accuracy, is the benefit of this technology, reinforced in recent systemic reviews and meta-analyses of robot-assisted screw placement compared to freehand placement. ${ }^{13,22}$ Arguably, it is the surgeon's skill, not the device, that delivers individual patient safety. However, as listed below, safety is a multistep 
process that is supported and enhanced by imageguided robotics:

(1) The preoperative planning software with its enhanced image processing, prepares the surgeon for the OR by presenting the abnormal scoliosis pedicle and vertebral morphology in 3 dimensions. ${ }^{1,2,23}$ This preview of the vertebral anatomy and simulated screw placement allows the surgeon to decide if the level should be instrumented by alternative fixation such as a hook or sublaminar band, or if the level should be abandoned altogether. The ability to create a CT-based implant strategy saves time in the OR, reduces implant costs, and increases patient safety by preventing repeated attempts to implant a screw into severely dysplastic anatomy.

(2) The device enables the surgeon to place the best-fitting pedicle screw at the best starting point with the correct trajectory in key anatomically feasible vertebra. Thus, the surgeon enhances patient safety through better control of the spine and decreased screw pullout and plow during correction maneuvers. Furthermore, repetitive planning of screw trajectories and sectional visualization of spinal anatomy improves the surgeon's 3D visualization skills over time and adds to their manual skills. This enhancement is in contrast to the common fear that continued reliance on image guidance would degrade the surgeon's spatial recognition and manual skills.

(3) Robotic surgical assistance improves patient safety by improving ergonomics and decreasing surgeon fatigue. Decreasing fatigue is especially important with severe complex deformities, high implant-density constructs, or multiple-case surgical days. Rather than focusing on screw placement, the surgeon is able to concentrate on corrective techniques and creating spinal balance. Also, consistent OR workflow contributes to a safer experience for the patient. All screw insertions take the same amount of time regardless of the anatomy. Screw sizes are known ahead of time and the implants are preselected, saving time. Finally, the robot dramatically reduces intraoperative fluoroscopy dosing to both the patient and operating team. ${ }^{22}$
As a newer technology, it is difficult to clearly define what the precise learning curve for imageguided robotics is; however, numerous advantages become apparent after the surgeon performs more cases. ${ }^{24}$ Although the initial disruption in the traditional surgical workflow may be misinterpreted as slow or inefficient, our data suggest that the surgical case duration soon returns to the previous baseline and then became quicker and more consistent with experience. Many surgeons are uncomfortable with "blind reliance" on the robotic stereotactic alignments, but the addition of navigation technology to the robotic arm and working tools adds reassurance and reduces fluoroscopic imaging for reviewing screw placement. Whereas the cost and maintenance of this enabling technology is high and is a barrier for some institutions, value can be ascertained by measuring clinical benefit against the cost. Thus, surgeons will be tasked with demonstrating improved clinical outcomes and OR efficiency with less revision surgery and radiation exposure.

Spinal robotic-assisted surgery is still in its nascent stage, and the innovative evolution of improvements is rapidly developing. As the state of the art moves from computer-assisted navigation with motion-controlled robotic stereotactics to a more autonomous form, inserting screws will be just one of the capabilities. Robotic-controlled facetectomies, bone removal, and osteotomies, and navigated interbody placement are on the forefront. Integration of magnetic resonance data rather than radiographic imaging is another possibility, along with expanding minimally invasive procedures to include deformity correction. Finally, one would expect cost reduction and miniaturization to follow a similar path as has occurred with computers and cell phones.

\section{REFERENCES}

1. Shoham M, Lieberman IH, Benzel EC, et al. Robotic assisted spinal surgery-from concept to clinical practice. Comput Aided Surg. 2007;12(2):105-115.

2. Devito DP, Kaplan L, Dietl R, et al. Clinical acceptance and accuracy assessment of spinal implants guided with SpineAssist surgical robot: retrospective study. Spine. 2010;35(24):2109-2115.

3. $\mathrm{Hu} \mathrm{X}$, Lieberman IH. What is the learning curve for robotic-assisted pedicle screw placement in spine surgery? Clin Orthop Relat Res. 2014;472(6):1839-1144.

4. Gertzbein SD, Robbins SE. Accuracy of pedicle screw placement in vivo. Spine. 1990;15(1);11-14.

5. Togawa D, Kayanja MM, Reinhardt MK, et al. Bone- 
mounted miniature robotic guidance for pedicle screw and translaminar facet screw placement: part 2 evaluation of system accuracy. Neurosurgery. 2007;60(2 suppl 1):129-139.

6. Pechlivanis I, Kiriyantham G, Engelhardt M, et al. Percutaneous placement of pedicle screws in the lumbar spine using a bone mounted miniature robotic system: first experiences and accuracy of screw placement. Spine. 2009; 34(4):392398.

7. Devito DP, Hegde SK, Woo R, Lieberman IH, Bederman SS. Retrospective analysis of feasibility and performance of robotic guidance for placement of pedicle screws in 223 adolescents with idiopathic scoliosis. Paper presented at: 21st International Meeting on Advanced Spine Techniques; July 1619, 2104; Valcencia, Spain.

8. Devito DP, Fernandez M, Blizzard DJ. Longitudinal assessment of robotic-assisted spine surgery: accuracy and improvements in efficiency. J Spine Surg. 2016;16(10):S369.

9. Shaw KA, Murphy JS, Devito DP. Accuracy of robot assisted pedicle screw insertion in AIS: is triggered EMG pedicle screw stimulation necessary? J Spine Surg. 2018;4(2):187-194.

10. Macke JJ, Woo R, Varich L. Accuracy of robot-assisted pedicle screw placement for AIS in pediatric population. $J$ Robot Surg. 2016;10(2):145-150.

11. Sensakoyc WF, O’Dell MC, Agha A, Woo R, Varich L. $\mathrm{CT}$ radiation dose reduction in robot assisted pediatric spinal surgery. Spine. 2016;42(7):417-424.

12. D'Souza M, Gendreau J, Feng A, et al. Robotic-assisted spine surgery: history, efficacy. cost, and future trends. Robotic Surg: Res Rev. 2019;6:9-13.

13. Fan Y, Du JP, Liu JJ, et al. Accuracy of pedicle screw placement comparing robot-assisted technology and the freehand with fluoroscopy-guided method in spine surgery. Medicine. 2018;97(22):e10970 doi:10.1097/MD.0000000000010 970

14. O'Connor TE, O'Hehir MM, Khan, A, et al. Mazor X Stealth robotic technology: a technical note. World Neurosurg. 2021;145:435-442. doi:10.1016/j.wneu.2020.10.010

15. Lieberman IH, Kisinde S, Hesselbacher S. Roboticassisted pedicle screw placement during spine surgery. JBJS Essen Surg Tech. 2020:10(2):e0020. doi:10.2106/JBJS.ST.19. 00020

16. Molliqaj G, Schatlo B, Alaid A, et al. Accuracy of robotguided versus freehand fluoroscopy-assisted pedicle screw insertion in thoracolumbar spinal surgery. Neurosurg Focus. 2017;42(5):e14. doi:10.3171/2017.3.FOCUS179

17. Schatlo B, Martinez R, Alaid A, et al. Unskilled unawareness and the learning curve in robotic spine surgery. Acta Neurochir. 2015;157(10):1819-1823.

18. Laratta JL, Shillingford JN, Lombardi JM, et al.
Accuracy of S2 alar-iliac screw placement under robotic guidance. Spine Deform. 2018;6(2):130-136.

19. Hu X, Lieberman I. Robotic-guided sacro-pelvic fixation using S2 alar-iliac screws: feasibility and accuracy. Eur Spine J. 2017;26(3):720-725.

20. Gonzalez D, Ghessese S, Cook D, Hedequist D. Initial intraoperative experience with robotic-assisted pedicle screw placement with stealth navigation in pediatric spine deformity: an evaluation of the first 40 cases. J Robotic Surg. 2021;15(5):687-693. doi:10.1007/s11701-020-01159-3

21. Hartl R, Lam KS, Wang J, et al. Worldwide survey on the use of navigation in spine surgery. World Neurosurg. 2013;70(1):162-172.

22. Fatima N, Massad E, Hadzipasic M, Shankar GM, Shin JH. Safety and accuracy of robot-assisted placement of pedicle screws compared to conventional free-hand technique: a systematic review and meta-analysis. Spine J. 2021;21(2):181192.

23. Kochanski RB, Lombardi JM, Laratta J, et al. Image guided navigation and robotics in spine surgery. Neurosurgery. 2019;84(6):1179-1189.

24. Khan A, Meyers JE, Siasios I, Pollina J. Next-generation robotic spine surgery: first report on feasibility safety, and learning curve. Oper Neurosurg. 2019;17(1):61-69.

Disclosures and COI: Dr Devito is on the Advisory Boards of Medtronic Spine and Stryker Spine; he is also a consultant for Astura Spine and SeaSpine. Dr Woo is a consultant for SeaSpine, Innovasis, and Implanet; he also receives royalties from SeaSpine and Innovasis.

Corresponding Author: Dennis P. Devito, MD, Medical Director, Spine Program, Children's Healthcare of Atlanta-Orthopedics and Sports Medicine, 5445 Meridian Mark Rd, Suite 250, Atlanta, GA 30342. Phone: (404) 255-1933; Email: dpdevito21@gmail.com.

\section{Published 26 October 2021}

This manuscript is generously published free of charge by ISASS, the International Society for the Advancement of Spine Surgery. Copyright (c) 2021 ISASS. To see more or order reprints or permissions, see http://ijssurgery.com. 